\title{
Effects of consuming sweeteners on metabolic disorders
}

\author{
Bruno S Lemos,' Maria-Luz Fernandez,' Maria Emilia S M Santos ${ }^{2}$ \\ 'Department of Nutritional Sciences, University of Connecticut, USA \\ ${ }^{2}$ Department of Biochemistry, Federal University of Triangulo Mineiro, Brazil
}

Correspondence: Bruno S Lemos, Department of Nutritional Sciences, University of Connecticut, USA, Email bruno.lemos@uconn.edu

Received: April 10, 2018 | Published: April 30, 2018

Copyright@ 2018 Lemos et al. This is an open access article distributed under the terms of the Creative Commons Attribution License, which permits unrestricted use, distribution, and reproduction in any medium, provided the original author and source are credited.

\begin{abstract}
Obesity is a prevalent health disease that has been associated with many metabolic disorders. As a risk for cardiovascular disease, the leading cause of death worldwide, dietary intervention or modifications are made to prevent or burden the effects of such pathology. On the other hand, added sugar is of great concern to the American population due to its association with diet induced obesity. Overconsumption of sweeteners is alarming since it can increase visceral fat, inflammation, and further lead to type II diabetes. The use of artificial sugar additives has increased and consequentlymore studies need to be done to explore adequate intake in various population groups, especially the diabetic. Ingestion of xylitol and erythritol, natural sweeteners, has gained popularity worldwide, yet not much is known about its metabolic effects and possible dosages that can be beneficial. Additional research needs to be done to explore the effectsof these sweeteners in the diabetic population.
\end{abstract}

\section{Introduction}

Obesity is an increasing epidemic of great health concern in the United States and worldwide. It estimated that obesity prevalence will be $33 \%$ by 2030 among Americans adults. ${ }^{1}$ Metabolic dysregulation can be caused by obesity which leads to a disruption in the various functions of the primarily metabolic organs: liver, adipose tissue, pancreas, muscle and intestines. ${ }^{2}$ Therefore, adipocyte hypertrophy caused by an unbalanced diet and an increase in caloric intake may mediate increased visceral fat and impaired adipose functionality., This will eventually result in macrophage infiltration to the tissue causing an increase in chemokine production, such as tumor necrosis factor. ${ }^{5}$ Insulin resistance (IR) caused by this inflammatory state can enhance lipolysis in the adipose tissue. ${ }^{6}$ Consequently, free fatty acids (FFA) will be released in the blood stream which can further contribute to another metabolic dysfunction, for example hepatic steatosis.

The liver is a major organ responsible for regulating many metabolic pathways. The FFA coming from the adipose tissue will overload the liver and this can contribute to hepatic steatosis, which is the accumulation of fat in the hepatocytes. ${ }^{2}$ Again, this will cause an increased production of pro-inflammatory cytokines and further enhance IR. The liver will start to make glucose via gluconeogenesis due to this IR. The excessive glucose in the blood can further be converted to lipids in the skeletal muscle and there accumulate, which will also contribute to IR. ${ }^{2}$ Overall these are contributing factors that will cause metabolic disease, specially referring to cardiovascular disease (CVD), type 2 diabetes (T2D), and non-alcoholic fatty liver disease (NAFLD).

Diet is a great contributor to metabolic functions. The role of energy intake versus energy expenditure is of great importance. Considering this concept, a balance among the intake of various macronutrients needs to be maintained not to cause increased energy accumulation in the fat form in the adipose tissue. With that, the controversy of sugar consumption continues due to its impact on health outcomes. The United States is the most consumers of added sugars, $15 \%$ in daily calories. $^{7}$ The World Health Organization recommends that added sugar should not exceed $10 \%$ of total caloric intake, similar guidelines from the American Heart Association in which suggests the intake of no more than 6 table spoon full ( 24 grams, equivalent to 100 calories) for women and 9 table spoon full for men(36 grams equivalent to 150 calories) ${ }^{8}$ NHANES data show that in the $21^{\text {st }}$ century the amount of sugar per year has reached 160 pounds per capita and that more than $40 \%$ of "sugars" added to the diet come from soft drinks and fruit drinks. The current increase in soft drink consumption in the United States is equivalent to 16 -oz per person per day. ${ }^{9}$ This review paper has the main objective to discuss the various types of added sugars that are currently used by consumers and in the food industry, along with their impact on metabolic disorders.

\section{Sucrose and high-fructose corn syrup}

With the increase in added sugar as well as many other nutrients in the United States, sucrose and high-fructose corn syrup (HFCS) have been target to be associated with the obesity epidemic. First of all, it is important to identify that sucrose is a disaccharide where fructose and glucose are bound..$^{10}$ On the other hand, HFCS has free fructose and glucose at various concentrations depending on the product and company that produced it. Since this topic is very controversial, understanding of the metabolism of these molecules after ingestion needs to be explained. Once sucrose is ingested, in the small intestines, the enzyme sucrase will break it down into fructose and glucose. ${ }^{10}$ Consequently, free fructose and glucose are absorbed. Additionally, 
studies have shown that in an acidic environment, for example carbonated soft drinks which are mildly acidic or high temperatures, can cause the bond in sucrose to break. ${ }^{10}$ Therefore, sucrose is likely to be already broken down into these two monosaccharides prior to consumption. Rippe et al. ${ }^{11}$ has proved in his laboratory that sucrose and HFCS behave identically based on his research data. Others disagree due to limitations of his studies, later discussed in this paper, along with biochemical pathways that fructose and glucose have in the liver.

Fructose is a simple sugar with a similar chemical formula to glucose. The difference among these sugars is in the presence of a keto group that is bound to the carbon 2 of fructose, while an aldehyde group at carbon 1 in the glucose molecule. ${ }^{12}$ The consumption of different amounts of these molecules may have an impact on the metabolic function. HFCS currently used in beverages contains 55\% fructose made by industrial enzymatic isomerization of the two molecules. ${ }^{12}$ Therefore, the excessive intake of fructose can activate fructose metabolism favoring lipogenesis in the liver that differs from glucose metabolism.

In the hepatocytes, both fructose and glucose are metabolized after ingestion. The liver is abundant in the glucose transporterGLUT5 that is responsible for the uptake of fructose to the cytoplasm of the cell. ${ }^{9}$ The first step in fructose metabolism is mediated by fructokinase, not regulated by energy status, where phosphorylation of fructose occurs by transferring a phosphorus group from ATP, this consequently produces a byproduct ADP which is later metabolized to uric acid. ${ }^{13}$ Afterwards, the phosphorylated fructose is further metabolized by aldolase that results in the trioses used for triglycerides (TG) backbone synthesis. ${ }^{9}$ Studies that observe an increase in post prandial triglycerides with acute or long term fructose-containing beverage feeding, is explained by this pathway where fructose accounts for increase in de novolipogenesis. ${ }^{9} \mathrm{On}$ the other hand, glucose enters the hepatocytes by GLUT2 and in other cells GLUT4 which is an insulin-dependent transporter. Once inside the cell, glucose is phosphorylated by glucokinase to form glucose-6-phosphate which is the regulatory step for glycolysis where now glucose is used as energy source to generate ATP.In case of excessive glucose, which is tightly regulated by phosphofructokinase, formation of triglycerides backbone occurs. ${ }^{12}$ Another important difference between fructose and glucose is that glucose transporters (GLUT1/3) are present in other tissues. ${ }^{14}$ For example, glucose enters beta pancreatic cells to trigger insulin secretion in response to increase glucose concentration in blood stream. ${ }^{12}$ The increase in circulating insulin can amplify satiety signals in the central nervous system, which fructose cannot since its transporters are not present in these tissues. ${ }^{10} \mathrm{The}$ most important point between the metabolism of this two molecules is that fructose facilitates the formation of triglycerides more efficiently than glucose, consequently it is more lipogenic. ${ }^{12}$ This can be critical for individuals with hyperlipidemia, IR, or T2D.

Insulin plays a major role regulating homeostasis in the body especially in response to elevated glucose concentrations. Insulin signals the central nervous system to promote satiety, and stimulates the synthesis and secretion of leptin. ${ }^{10}$ Leptin is a hormone produced by the adipose tissue responsible for regulating long-term homeostasisacting in the hypothalamus to regulate food intake and energy metabolism. ${ }^{12}$ Consequently, the decrease in insulin response and leptin production caused by an increase in fructose-containing foods may lead to long-term deleterious effects on the regulation of energy intake and body adiposity. While glucose increases the "satiety" signaling by regulating insulin, leptin and ghrelin, studies have shown that fructose, due to its moiety, interacts differently and does not regulate these energy hormones which eventually causes excess calories to be consumed.$^{10}$ This can increase the chances of weight gain and obesity, which further contribute to metabolic dysfunction.

Additionally, sucrose and fructose containing foods are primarily in liquid form versus solid. Recent studies have shown that satiation is less between soft drink calories and solid food. ${ }^{11}$ Studies have shown that liquid sugar may promote an increase energy intake in comparison to solid foods, especially fructose containing foods, ${ }^{8}$ and may cause weight gain since sugar in liquid is digested quicker and can promote greater substrate overload to the liver. ${ }^{7}$ To date, it has been observed that the fructose metabolism induce non enzymatic glycation which is linked to oxidative stress and inflammation. ${ }^{8}$ These two factors play an important role in the development of metabolic diseases. Importantly, sugar consumed at high amounts are considered "toxic" and it is metabolized in the liver to fat which can further aggravate the risk form CVD, obesity, T2M, NAFLD and hypertension.

Fructose metabolism is responsible for depletion of ATP in the liver which increases the degradation of nucleotides to uric acid, which can be a contributor factor for elevated blood pressure. ${ }^{13}$ Studies have shown that increased uric acid concentration has been associated with chronic kidney diseases and CVD. ${ }^{12}$ Additionally, uric acid has been shown to cause endothelial dysfunction that could increase blood pressure. ${ }^{10}$ Meta-analysis has reported that consumption of 17.5 or $25 \%$ of daily added sugars as HFCS increase fasting and 24-hour uric acid levels. ${ }^{7}$

Fructose and glucose are rarely consumed isolated in the human diet. Therefore, studies using pure fructose and glucose specially in animal models needs to be interpreted with caution when translating the results to humans. ${ }^{13}$ One group has reported that individuals consuming fructose-sweetened beverages for 10 weeks showed increased de novolipogenesis (DNL), dyslipidemia, and circulating uric acid levels while reduced fatty acid oxidation and insulin sensitivity in comparison to subjects consuming glucose-sweetened beverages. ${ }^{7}$ The increase in DNL promotes production and secretion of very low density lipoprotein (VLDL) which is responsible for the increase in post prandial triglycerides and dyslipidemia. ${ }^{7}$ With increasing inflammation and oxidative stress, a cascade of events contributes to an increase in hepatic lipid, which will reduce liver insulin sensitivity and can further increase DNL. ${ }^{13}$ Eventually, an increase in apolipoprotein (apo) B (apoB) availability (VLDL backbone) can be observed, with an up regulation of microsomal triglyceride-transfer protein expression (protein responsible for assembling TG and apoB in VLDL particle) and increase production of apoCIII. Importantly apoCIII has been shown to now only promote lipid poor VLDL to TG-rich LDL, but it is also linked to hypertriglyceridemia due to inhibition of lipoprotein lipase activity and clearance of VLDL by the liver. ${ }^{7}$ Thus, increased levels of apoCIII becomes a strong biomarker of independent pathways for CVD risk. Other studies evaluated the consumption of 1 liter/day of sucrose-sweetened cola for 6 months and observed an increase in liver TG and fasting plasma TG in comparison to a isocaloric low-fat milk. ${ }^{7}$ Another group reported that young, healthy men consumed $80 \mathrm{~g}$ /day sucrose in beverage for 3 weeks in comparison to the same amount of glucose, and an increase in low lipoprotein cholesterol (LDL-C) and small LDL-C was observed. LDL-C is a biomarker for CVD and small LDL-C can beoxidized 
which is a major leading factor to atherogenesis. Limitations of these studies are the total amount of sugar consumed by the participants is unknown since they were adding the intervention to their habitual diet. Another study where participants consuming HFCS or sucrose equivalent to 40,90 , or $150 \mathrm{~g}$ of sugar per day in a 2000 -kcal diet for 10 week period using a low-fat milk as a vehicle did not observe any significant difference between baseline and end of intervention. ${ }^{10}$ Unfortunately, the use of low fat milk could have been a confounder in this study, since low fat milk has been previously shown to decrease triglyceride, due to milk proteins and lipids interference with intrahepatic lipids and alteration of lipoprotein kinetics. ${ }^{10}$ In contrast a group that conducted a study using sweetened beverages with increasing doses of $\operatorname{HFCS}(0,10,17.5$, or $25 \%)$ using water and not milk for 2 weeks, observed an increase in a dose dependent manner for TG, LDL-C, apoB, apoCIII, and uric acid. ${ }^{15}$

Importantly, the excessive intake of sucrose and HFCS may disrupt metabolic functions and increase risk of metabolic diseases. Analysis on availability of HFCS and prevalence of diabetes in countries has an great association, showing more than $20 \%$ prevalence of diabetes than countries that do not use HFCS. ${ }^{16}$ Naturally occurring fructose, found in fruits and vegetables, can have beneficial effects due to protective effects against diabetes, but the amount of consumed fructose is the major concern. ${ }^{8}$ Added sugars need to be consumed with caution. Therefore, the awareness to carefully control the diet is important in order to monitor the amount of added sugars.

\section{Artificial sweeteners}

Classification of sweeteners are based on the sweet intensity and nutritive value, it includes the caloric added sugars previously described and the non-caloric high intensity that are most commonly known as artificial sweeteners. Thus far, the food industry uses mainlyaspartame, saccharin, sucralose and acesulfame potassium. Aspartame is composed of phenylalanine and aspartate that are linked through a methanol backbone, and it is 200 time sweeter than sucrose. Its safety is questioned due to the metabolites formed after ingestion: formaldehyde, formic acid and diketopiperazine. ${ }^{8} \mathrm{On}$ the other hand, saccharin is 300 times sweeter than sucrose and it is considered the oldest artificial sweeteners discovered. Sucralose is the sweetest sweetener synthesized from sucrose, it is a disaccharide where the three hydroxyl groups from the sucrose are substituted for three chlorine groups. ${ }^{8}$ Lastly, acesulfame potassium is a heat stable, moderate sweet (120 times than sucrose) sweetener used for cooking and baking. ${ }^{8}$ There is ongoing research to investigate the effects of these artificial sweeteners of metabolic diseases.

Since obesity is an alarming prevalent disease, cohort studies have evaluated the effects of consuming artificial sweeteners with weight gain. The San Antonio Heart Study observed that consuming artificially sweetened beverages versus none,for more than 21 days doubled the chances of being overweight or obese. ${ }^{8}$ Another American Cancer Society study, evaluated that regardless of initial BMI, during an one year follow up, artificial sweetened beverage consumers were more likely to gain weight in comparison to nonconsumers. ${ }^{8}$ Researchers believe that the sweetness in these drinks favors an incomplete activation of the food reward pathway, therefore causing the consumers to seek more caloric food as well as encourage sweet cravings. ${ }^{8}$ Other cohort studies found an association between consumption of artificial sweetened beverages and metabolic diseases such as metabolic syndrome, type 2 diabetes, hypertension and cardiovascular disease. ${ }^{17}$ To support these cohort studies, an experiment with normal weight adult males consuming aspartatesweetened water, or other artificial sweeteners such as acesulfame potassium or saccharin, results in an increased appetite and motivation to eat in these individuals. Similar effects were observed in rats treated with saccharin supplementation, showing increased energy intake and weight gain. ${ }^{8}$ This effect can be further explained by the fact that artificial sweeteners stimulate food intake because of a reduction in the sweet tastes. Sweet tastes are physiological responses that help regulate energy homeostasis caused by the signals from nutrients in the gut in order to facilitate absorption and utilization of energy foods. ${ }^{17}$ In an animal model, following the consumption of a sucralose solution or aspartame sweetened beverage, release of hormones and markers (insulin, glucagon-like peptide-1 [GLP-1], peptide YY [PYY], and ghrelin) for post-prandial glucose homeostasis was not observed, which further explains the metabolic and hormonal effects of these added sugars. ${ }^{17}$ Artificial sweeteners seem to firstly affect satiety in order to drive an increased eating behavior favoring consumption of high caloric sugar foods.

Even though these artificial sweeteners have effects on metabolic functions, precautions need to be taken for those that consume them excessively. The Food and Drug Administration has classified the artificial sweeteners are safe based on scientific evidence. Saccharin has been given an acceptable daily intake (ADI) of $5 \mathrm{mg} / \mathrm{kg} /$ day, and it is known that after oral ingestion this disaccharide is neither absorbed nor metabolized since it is excreted in the urine unchanged. ${ }^{18}$ Sucralose is the sweetest artificial sweetener but it has the same ADI as saccharin because it is also secreted unchanged in the feces. ${ }^{18}$ On the other hand, aspartame has ADI of $50 \mathrm{mg} / \mathrm{kg} /$ day which is hydrolyzed upon ingestion into the two amino acids and methanol, and these metabolites can be further metabolized. ${ }^{18}$ Lastly, acesulfame potassium has ADI of $15 \mathrm{mg} / \mathrm{kg} /$ day that is rapidly absorbed after ingestion and excreted unchanged through the urine. ${ }^{18}$ The majority of the non-caloric artificial sweeteners act by inhibiting the sweet tastes causing an increased consumption of caloric foods, therefore resulting in the possible imbalance of the energy homeostatic pathway.

\section{Xylitol and erythritol}

With the emerging use of added sugars around the world, xylitol and erythritol have become very popular, especially for the diabetic population. Both these sugars are found naturally in fruits and vegetables such as strawberries, raspberries, plums and lettuce. ${ }^{19}$ Xylitol is a five carbon alcohol sugar that has lower glycemic index than sucrose (13 versus 65$)$ and caloric value (2.4 versus $4.0 \mathrm{kcal} /$ gram), which makes it a great alternative for diabetics. ${ }^{20}$ On the other hand, erythritol is a four carbon polyol with a sweetness of about $60-80 \%$ of sucrose. ${ }^{21}$ The metabolism of these two sweeteners vary and that is a great determinant of their effects on metabolic disorders. For instance, xylitol is partially absorbed in the stomach and upper gastrointestinal tract, which is later metabolized in the liver by oxidation to D-xylulose. ${ }^{19}$ This last product can now enter the pentose phosphate pathway which a majority will later form glucose, or small amount will be converted to lactate. ${ }^{19}$ Erythritol, similar to the artificial sweeteners, is efficiently absorbed but not metabolized, and will later be excreted unchanged in the urine. ${ }^{21}$ These sweeteners are considered safe for diabeticstaking in account the previous mentioned alternatives, and xylitol is rapidly absorbed and cleared, therefore not accumulating in tissues. ${ }^{22}$ 


\section{Xylitol effects on metabolic organs}

Type 2 diabetes is a metabolic disorder resulting in hyperglycemia that involves IR accompanied by pancreatic beta-cell dysfunction. ${ }^{20}$ Xylitol has been shown to be a great alternative for diabetic patients. It is effective in reducing visceral fat which is one of the factors contributing to IR and further progression of T2D. ${ }^{23}$ When feeding animals with xylitol $(1-2 \mathrm{~g} / 100 \mathrm{kcal})$ for 8 weeks, visceral fat mass was lower and lipid metabolism was affected. ${ }^{3}$ Amo et al. ${ }^{3}$ showed that xylitol intake will be absorbed in the intestines by passive diffusion and then metabolized to xylulose 5-phosphate (Xu5P) in the liver. Xu5P activates carbohydrate response element binding protein (ChREBP) which will activate lipogenic genes such as coenzyme A carboxylase and fatty acid synthase. ${ }^{24,25} \mathrm{On}$ the other hand, oral xylitol decreased plasma insulin while increasing insulin sensitivity with the observed increase in adiponectin. It is not yet known how xylitol affect the adipose tissue since most of it is metabolized by the liver, but an increase in expression of peroxisome proliferator-activated receptor (PPAR) gamma (PPAR $\gamma$ ) was observed in the adipose tissue which up regulates adiponectin expression as well as lipolysis. ${ }^{3}$ With the decrease in IR, an increase in hepatic PPAR $\alpha$ was seen, which is responsible for fatty acid oxidation. These mechanisms explain how the balance in lipolysis and lipogenesis between the metabolic organs, prevented the progression of visceral fat accumulation which is one of the factors contributing to T2D and CVD.

Currently, emerging research has been focusing on the association of the gut microbiota and metabolic disorders. Importantly, microorganisms in the gut utilize nutrients as substrates to produce metabolites that can either be beneficial or detrimental that modulate host metabolic functions. ${ }^{26}$ Dietary fiber is fermented by the gut microbiota to produce short chain fatty acids (SCFA) such as butyrate, propionate and acetate; while other nutrients such as choline is metabolized to produce trimethylamine (TMA). ${ }^{26}$ These metabolites can function as signals or be further oxidized, in the case of TMA. SCFA has shown to signal to induce the expression of PYY and GLP-1, while TMA in metabolized to trimethylamine-N-oxide (TMAO) in the liver. ${ }^{26,27}$ In fact, TMAO has been shown to be associated with atherosclerosis progression. ${ }^{28}$ Low (40 mg/kg body weight) and medium $(194 \mathrm{mg} / \mathrm{kg}$ body weight) doses of xylitol in a mice model have shown to gradually modulate the gut microbiota composition. A decrease in Bacteroidetes phylumand Barnesiellagenuswas observed with the medium dose with either control of high fat diet. ${ }^{29}$ Interestingly, Bacteroidetesare TMA producers using L-carnitine as a substrate, which can further increase TMAO plasma concentrations. ${ }^{30}$ Additionally, $5 \%$ xylitol in a diet had also decreased Bacteroidetesin mice, gram-negative bacteria, and a similar shit from these gram-negative to gram-positive bacteria was observed in humans at a $30 \mathrm{~g}$ oral dose of xylitol. ${ }^{31,32} \mathrm{On}$ the other hand, medium dose of xylitol increased Firmicutesphylumand Prevotellagenus and ratio of Prevotella to Bacteroidetes. ${ }^{29}$ Abundance of Prevotella has been associated with improved glucose metabolism, and Firmicutes have shown to accelerate food degradation resulting in higher energy supply to the host. ${ }^{33}$ Lastly, xylitol has shown to suppress lipopolysaccharide induce inflammation in a chicken model. Therefore, xylitol intake can modulate the gut microbiota composition which will further affect metabolic functions.

\section{Recent research and future directions}

Thus far is not know the right dose of xylitol that is harmful or has the optimal effect in diabetic complications related to T2D. A dosedependent study done in animals, evaluated three different dosages in diabetic rats $(2.5,5.0$, or $10 \%)$, where the highest dose showed to be the most effective in reducing blood glucose and serum fructosamine levels. ${ }^{23}$ Additionally, beneficial changes in the morphology of pancreatic beta cells was observed with the $10 \%$ dose including increasing in size of islets and number of cells. ${ }^{23}$ Other studies evaluated the effects of xylitol, in vivoand ex vivo, in improving circulating glucose by promoting the glucose uptake in the muscle and fat cells which would ameliorate hyperglycemia in diabetics. ${ }^{20}$ On the other hand, erythritol, even though it is not metabolized and is efficiently excreted, has shown to be an excellent hydroxyl radical scavenger. ${ }^{21}$ This property is extremely important, especially in diabetic patients, because chronic hyperglycemia can lead to inflammation which will further aggravate endothelial dysfunction due to increased oxidative stress, a factor associated with cardiovascular diseases. ${ }^{21}$ Therefore, this antioxidant effect attributed to erythritol was evaluated in an endothelial cell line showing that the damage done by oxidative stress and free radicals generated in response to elevated glucose was reduced with erythritol treatment. ${ }^{21}$ Along with these results, other studies in humans where done to evaluate effects of xylitol. One study were participants consumed $25 \mathrm{~g}$ of xylitol in $300 \mathrm{~mL}$ of water prior to a solid meal, resulted in prolonged gastric emptying and decreased food intake. ${ }^{34}$ Another study, with $30 \mathrm{~g}$ of xylitol in $200 \mathrm{~mL}$ of water prior to a meal showed enhanced intestinal nutrient transit in comparison to glucose ${ }^{23}$ Again, the effects of these added sweet alternatives are attributed to the sweet taste receptors. In the case of xylitol and erythritol, both stimulated the secretion of GLP1 , a marker for the activation of the sweet receptors in the gut, in a study with healthy and obese patients..$^{34}$ In response to this incretin, controlled eating behavior and energy homeostasis regulation was observed. In regards to prolonged gastric emptying, it is important because it leads to a feeling of fullness and satiation, which consequently results in termination of a meal. This phenomenon is caused by a feedback loop from GLP-1 and CCK (cholecystokinin), which were both increased after treatment with xylitol and erythritol. ${ }^{34}$ Of course, more research needs to be carried out further because there are side effects associated with the intake of these sweeteners such as diarrhea, bloating, nausea and borborygmic. ${ }^{34}$ Nevertheless, natural occurring sweeteners are a promising alternative that should be used by consumers and the food industry.

\section{Conclusion}

With the increasing epidemic in obesity, other metabolic diseases emerge resulting in further complications and prevalence of pathologies. Along with this outbreak, consumption of added sugars has been associated as a great factor for the development of such diseases. An alternative and beneficial option is using natural occurring sweeteners that have shown to be highlysoluble, containing an antioxidant activity and helping regulate energy intake homeostasis. Further research needs to be done to understand the mechanisms and doses of these sweeteners in various population, especially in diabetic patients with great focus on the gut microbiota since it plays a major role in the host metabolism.

\section{Conflict of interest}

The authors wish to declare no conflict of interest. 


\section{References}

1. Thomas DM, Weedermann M, Fuemmeler BF, et al. Dynamic Model Predicting Overweight, Obesity, and Extreme Obesity Prevalence Trends. Obesity(Silverspring). 2014;22(2):590-597.

2. Lackey DE, Olefsky JM. Regulation of metabolism by the innate immune system. Nat Rev Endocrinol. 2015;12:15.

3. Amo K, Arai H, Uebanso T, et al. Effects of xylitol on metabolic parameters and visceral fat accumulation. J Clin Biochem Nutr. 2011;49(1):1-7. doi:10.3164/jcbn. 10

4. Strable MS, Ntambi JM. Genetic control of de novo lipogenesis: role in diet-induced obesity. Crit Rev Biochem Mol Bio. 2011;45(3):199-214

5. Moore K, Sheedy F, Fished E. Macrophages in atherosclerosis : a dynamic balance. Nat Rev Immunol. 2015;13(10):709-721.

6. Freitas Lima LC, Braga V de A, do Socorro de França Silva M, et al. Adipokines, diabetes and atherosclerosis: An inflammatory association. Front Physiol. 2015;6(NOV):1-15.

7. Stanhope KL. Sugar consumption, metabolic disease and obesity: The state of the controversy. Crit Rev Clin Lab Sci. 2016;53(1):52-67.

8. Mooradian AD, Smith M, Tokuda M. The role of artificial and natura sweeteners in reducing the consumption of table sugar: A narrative review. Clin Nutr ESPEN. 2017;18:1-8.

9. Bray GA. Energy and Fructose From Beverages Sweetened With Sugar or High-Fructose Corn Syrup Pose a Health Risk for Some People. Adv Nutr Int Rev J. 2013;4(2):220-225.

10. Yu Z, Lowndes J, Rippe J. High-fructose corn syrup and sucrose have equivalent effects on energy-regulating hormones at normal human consumption levels. Nutr Res. 2013;33(12):1043-1052.

11. Rippe J, Etherton P. Fructose, sucrose, and high fructose corn syrup: modern scientific findings and health implications. Adv Nutr Int .... 2012:739-740.

12. Ferder L, Ferder MD, Inserra F. The role of high-fructose corn syrup in metabolic syndrome and hypertension. Curr Hypertens Rep. 2010;12(2):105-112.

13. Rippe JM, Angelopoulos TJ. Sucrose, High-Fructose Corn Syrup, and Fructose, Their Metabolism and Potential Health Effects: What Do We Really Know? Adv Nutr Int Rev J. 2013;4(2):236-245.

14. Meyers AM, Mourra D, Beeler JA. High fructose corn syrup induces metabolic dysregulation and altered dopamine signaling in the absence of obesity. PLoS ONE. 2017;12(12):8-11.

15. Stanhope KL, Medici V, Bremer AA, et al. A dose-response study of consuming high-fructose corn syrup - sweetened beverages on lipid / lipoprotein risk factors for cardiovascular disease in young adults 1-6. Am J Clin Nutr. 2015;101:1144-1154.

16. Goran MI, Ulijaszek SJ, Ventura EE. High fructose corn syrup and diabetes prevalence: A global perspective. Glob Public Health. 2013;8(1):55-64.

17. Swithers SE. Artificial sweeteners produce the counterintuitive effect of inducing metabolic derangements. Trends Endocrinol Metab. 2013;24(9):431-441.
18. Van Eyk AD. The effect of five artificial sweeteners on Caco-2, HT-29 and HEK-293 cells. Drug Chem Toxicol. 2015;38(3):318-327.

19. Schmid RD, Hovda LR. Acute Hepatic Failure in a Dog after Xylitol Ingestion. J Med Toxicol. 2016;12(2):201-205.

20. Chukwuma CI, Islam MS. Effects of xylitol on carbohydrate digesting enzymes activity, intestinal glucose absorption and muscle glucose uptake: a multi-mode study. Food Funct. 2015;6(3):955-962.

21. Boesten DMPHJ, Berger A, de Cock P, et al. Multi-Targeted Mechanisms Underlying the Endothelial Protective Effects of the Diabetic-Safe Sweetener Erythritol. PLOS ONE. 2013;8(6).

22. Peterson ME. Xylitol. Top Companion Anim Med. 2013;28(1):18-20.

23. Rahman MA, Islam MS. Xylitol Improves Pancreatic Islets Morphology to Ameliorate Type 2 Diabetes in Rats: A Dose Response Study. J Food Sci. 2014;79(7):1436-1442.

24. Ishii S, IIzuka K, Miller BC, Uyeda K. Carbohydrate response element binding protein directly promotes lipogenic enzyme gene transcription. Proc Natl Acad Sci. 2004;101(44):15597-15602.

25. Iizuka K, Bruick RK, Liang G, Horton JD, Uyeda K. Deficiency of carbohydrate response element-binding protein (ChREBP) reduces lipogenesis as well as glycolysis. Proc Natl Acad Sci U S A. 2004;101(19):7281-7286.

26. Schroeder BO, Bäckhed F. Signals from the gut microbiota to distant organs in physiology and disease. Nat Med. 2016;22(10):1079-1089.

27. Tang WHW, Hazen SL. The contributory role of gut microbiota in cardiovascular disease. J Clin Invest. 2014;124(10).

28. Wang Z, Klipfell E, Bennett BJ, et al. Gut flora metabolism of phosphatidylcholine promotes cardiovascular disease. Nature. 2011;472(7341):57-63.

29. Uebanso T, Kano S, Yoshimoto A, et al. Effects of consuming xylitol on gut microbiota and lipid metabolism in mice. Nutrients. 2017;9(7).

30. Koeth RA, Wang Z, Levison BS, et al. Intestinal microbiota metabolism of L-carnitine, a nutrient in red meat, promotes atherosclerosis. Nat Med. 2013;19(5):576-585.

31. Tamura M, Hoshi C, Hori S. Xylitol affects the intestinal microbiota and metabolism of daidzein in adult Male mice. Int $\mathrm{J} \mathrm{Mol} \mathrm{Sci}$. 2013;14(12):23993-24007.

32. Salminen S, Salminen E, Koivistoinen P, Bridges J, Marks V. Gut microflora interactions with xylitol in the mouse, rat and man. Food Chem Toxicol. 1985;23(11):985-990.

33. Khan MJ, Gerasimidis K, Edwards CA, Shaikh MG. Role of Gut Microbiota in the Aetiology of Obesity: Proposed Mechanisms and Review of the Literature. J Obes. 2016;2016.

34. Wölnerhanssen BK, Cajacob L, Keller N, et al. Gut hormone secretion, gastric emptying, and glycemic responses to erythritol and xylitol in lean and obese subjects. Am JPhysiol - Endocrinol Metab. 2016;310(11):E1053E1061 